\title{
Evolution and emergence of multidrug-resistant Mycobacterium tuberculosis in Chisinau, Moldova
}

\author{
Tyler S. Brown', Vegard Eldholm², Ola Brynildsrud², Magnus Osnes², Natalie Levy³, James Stimson4, Caroline Colijn ${ }^{5}$, \\ Sofia Alexandru ${ }^{6}$, Ecaterina Noroc ${ }^{6}$, Nelly Ciobanu ${ }^{6}$, Valeriu Crudu ${ }^{6}$, Ted Cohen ${ }^{7}$ and Barun Mathema ${ }^{3, *}$
}

\begin{abstract}
The evolution and emergence of drug-resistant tuberculosis (TB) has been studied extensively in some contexts, but the ecological drivers of these two processes remain poorly understood. This study sought to describe the joint evolutionary and epidemiological histories of a novel multidrug-resistant Mycobacterium tuberculosis strain recently identified in the capital city of the Republic of Moldova (MDR Ural/4.2), where genomic surveillance of drug-resistant M. tuberculosis has been limited thus far. Using whole genome sequence data and Bayesian phylogenomic methods, we reconstruct the stepwise acquisition of drug resistance mutations in the MDR Ural/4.2 strain, estimate its historical bacterial population size over time, and infer the migration history of this strain between Eastern European countries. We infer that MDR Ural/4.2 likely evolved (via acquisition of rpoB S450L, which confers resistance to rifampin) in the early 1990s, during a period of social turmoil following Moldovan independence from the Soviet Union. This strain subsequently underwent substantial population size expansion in the early 2000s, at a time when national guidelines encouraged inpatient treatment of TB patients. We infer exportation of this strain and its isoniazid-resistant ancestral precursor from Moldova to neighbouring countries starting as early as 1985 . Our findings suggest temporal and ecological associations between specific public health practices, including inpatient hospitalization of drug-resistant TB cases from the early 2000s until 2013, and the evolution of drug-resistant M. tuberculosis in Moldova. These findings underscore the need for regional coordination in TB control and expanded genomic surveillance efforts across Eastern Europe.
\end{abstract}

\section{DATA SUMMARY}

Whole genome sequencing data for isolates collected in Chisinau, Moldova are available in the National Centre for Biotechnology Information Sequence Read Archive (NCBI SRA bioproject number: PRJNA482716). Whole genome sequence data for the regional collection of isolates from Eastern Europe are available under NCBI SRA bioproject numbers PRJNA421446, PRJNA429460, PRJNA384815, PRJNA318002, PRJNA480888, and PRJNA482865.

\section{BACKGROUND}

Drug-resistant tuberculosis (TB) remains an important priority in global public health [1]. Drug-resistant tuberculosis, including multidrug-resistant (MDR) and extensively drugresistant (XDR) forms, are highly endemic in Eastern Europe and countries of the Former Soviet Union (FSU). In Moldova, which records the highest per-capita rates of MDR-TB worldwide ( 54 cases per 100000 ), nearly $25 \%$ of MDR-TB occurs among individuals without previous history of TB therapy and $>50 \%$ of previously treated TB cases are MDR [2].

Received 19 February 2021; Accepted 04 June 2021; Published 25 August 2021

Author affiliations: ${ }^{1}$ Infectious Disease Division, Massachusetts General Hospital, Boston, MA, USA; ${ }^{2}$ Division of Infectious Disease Control, Norwegian Institute of Public Health, Oslo, Norway; ${ }^{3}$ Department of Epidemiology, Mailman School of Public Health, Columbia University, New York, NY, USA; ${ }^{2}$ National Infection Service, Public Health England, London, UK; ${ }^{5}$ Department of Mathematics, Simon Fraser University, Vancouver, Canada; ${ }^{6}$ Phthisiopneumology Institute, Chisinau, Republic of Moldova; ' Department of Epidemiology (Microbial Diseases), Yale University School of Public Health, New Haven, CT, USA. *Correspondence: Barun Mathema, bm2055@cumc.columbia.edu

Keywords: antimicrobial resistance; epidemiology; Mycobacterium tuberculosis; outbreaks; phylogenomics.

Abbreviations: DTA, discrete trait analysis; MDR, multidrug-resistant; TB, tuberculosis; TMRCA, time to most recent common ancestor; WGS, whole genome sequencing: XDR, extensively drug resistant.

Whole genome sequence data used in this manuscript are available under National Centre for Biotechnology Information Sequence Read Archive Bioproject Numbers: PRJNA482716, PRJNA421446, PRJNA429460, PRJNA384815, PRJNA318002, PRJNA480888, and PRJNA482865.

Data statement: All supporting data, code and protocols have been provided within the article or through supplementary data files. Six supplementary figures and two supplementary tables are available with the online version of this article 000620 ๑ 2021 The Authors 
Drug-resistant TB cases in most high burden countries are attributable to ongoing transmission of specific, epidemiologically successful Mycobacterium tuberculosis (M. tuberculosis) strains, rather than de novo acquisition of drug resistance mutations while on anti-TB therapy [3-5]. A large body of existing research has examined how social and economic turmoil, health systems failures, and mass incarceration following the collapse of the Soviet Union likely facilitated both selection for and transmission of drug-resistant TB in Eastern Europe [6]. However, evidence linking specific historical events or public health practices to the emergence of drug-resistant $M$. tuberculosis strains is limited, particularly in heavily impacted countries like Moldova. This study reconstructs the evolutionary history of a recently identified [7], highly successful strain of MDR-TB currently circulating in Chisinau, Moldova (referred to here as Ural/4.2).

In this report, we employ whole genome sequencing (WGS) data to examine the genomic epidemiology of $M$. tuberculosis among a cross-sectional survey of TB patients diagnosed at a municipal hospital in Chisinau, Moldova. We find a high prevalence of MDR-TB and uncover the local predominance of a unique $M$. tuberculosis strain responsible for $>60 \%$ of MDR-TB cases in this sample. Using phylodynamic methods, we examine the joint evolutionary and epidemiologic histories of this strain in Moldova and surrounding countries, yielding new evidence on how specific public health practices facilitated the emergence and subsequent dispersal of this important public health threat. These results support the expanded use of genomic surveillance in local and regional TB control efforts.

\section{METHODS}

\section{Study site and participants}

The study population included 404 individuals with culturepositive TB at the time of initiation of inpatient treatment at a municipal TB hospital in Chisinau, Republic of Moldova from October 2013 to December 2014. This TB hospital serves the municipality of Chisinau (population approximately 700000). Male and female TB patients 5 years of age or over, both HIV negative and positive, were eligible for the study if they were able to provide informed consent and had a minimum of one positive M. tuberculosis culture result. Patients were excluded from the study if the quantity of respiratory secretions provided was less than $5 \mathrm{ml}$, if their $M$. tuberculosis culture was negative, or if informed consent not provided. Viable serial isolates were processed for whole genomic sequence analysis using methods described below. Additional information on the study site and participant metadata are included in the Supplementary Information.

\section{Ethical review}

The study protocol was approved by the Institutional Ethical Committee for Research of Phthisiopneumology Institute, Chisinau, Republic of Moldova, Yale School of Public Health, and Columbia University Medical Centre.

\section{Impact Statement}

This study uses whole genomic sequence data to examine the joint evolutionary and epidemiological history of a successful multidrug-resistant Mycobacterium tuberculosis (Mtb) strain in Moldova, where existing genomic data on $M$ tb is relatively sparse. This study adds new insights into the stepwise acquisition of antimicrobial resistance in this strain and highlights ongoing, recent transmission as an important driver of drug-resistant TB cases in Moldova. This work is of interest to infectious disease epidemiologists, clinicians, and decision-makers in public health and TB control programmes interested in surveillance and control of drug-resistant TB.

\section{Genomic characterization of $M$. tuberculosis clinical isolates from the Republic of Moldova}

Of 404 patient isolates collected in Chisinau, Moldova and sequenced via paired-end sequencing on the Illumina MiSeq platform, we recovered 376 (93\%) M. tuberculosis isolates with WGS data meeting criteria for read depth (average read depth $>15$ reads) and sequence coverage (reads covering $>99 \%$ of the reference genome). For phylogenetic analyses, we removed duplicate (serial) isolates by selecting, for each participant, the isolate with the earliest collection date, yielding a subset of $n=283$ non-duplicate individual isolates collected in Chisinau for analysis. Additional information on read alignment, filtering, and variant detection is included in the Supplementary Information.

\section{WGS data for Eastern European and lineage- representative $M$. tuberculosis isolates}

To provide regional context for the isolates collected in Chisinau, we obtained raw paired-end read data for $n=1168$ samples included in the NCBI Sequence Read Archive, including those from a publicly-available collection of $M$. tuberculosis genome sequence data from Eastern Europe [8] and well-characterized isolates from relevant $M$. tuberculosis phylogeographic lineages to anchor important clades in the phylogenetic analysis. Publicly available $M$. tuberculosis sequence data was obtained from TB Portals, a large regional TB data repository [8] that includes clinical cases and M. tuberculosis isolates from Moldova, Azerbaijan, Belarus, Georgia, and Romania; we obtained a convenience sample of all $M$. tuberculosis isolates with whole genome sequencing data available at the time of our analysis. Read filtering, alignment, and variant calling and filtering for these additional sequences were conducted following the procedures described above.

\section{Phylogenetic analysis}

We conducted phylogenetic analysis on three groups of M. tuberculosis isolates, doing model testing in BEAST2 [9], phylogenetic reconstruction and discrete trait analysis in BEAST 1.10 [10], and the IQtree and timetree tools available 


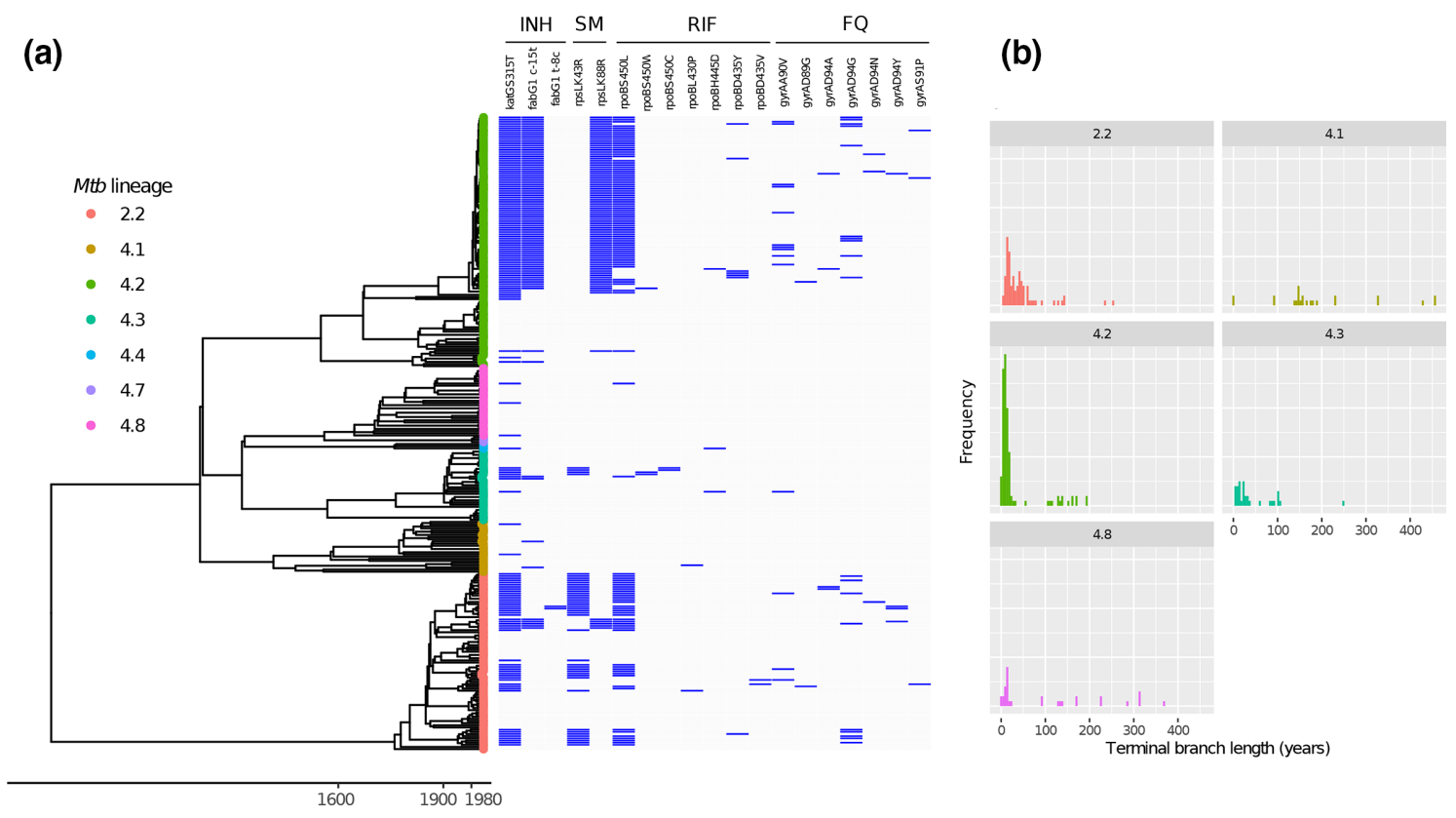

Fig. 1. Bayesian phylogenetic analysis for $n=293$ M. tuberculosis isolates collected in the Republic of Moldova in 2013-2014. (a) Dated phylogenetic reconstruction for all $n=293$ sequences collected in Chisinau and included in phylogenetic analysis. Tips of the tree are annotated by SNP barcode-based sublineage assignment [12]. Selected mutations associated with antimicrobial resistance (specifically those associated with resistance to isoniazid, streptomycin, rifampin, and fluoroquinolones) are annotated in the adjacent heatmap. (b) Distribution of terminal branch lengths (in years) by sublineage. Lineages 4.4 and 4.7 are not included in panel (b) given the small number of sequences in each clade. The phylogenetic tree was estimated using BEAST v1.10 with the HKY nucleotide substitution model, constant-size coalescent population model, strict molecular clock, and an informative prior on the mutation rate (Table S1).

through Nextstrain Augur version 6.2.0 [11] (Supplementary Methods and Table S1, available in the online version of this article).

\section{RESULTS}

\section{High prevalence of MDR-TB in the Republic of Moldova}

Our cross-sectional study of $M$. tuberculosis genome sequences collected from October 2013 - December 2014 in Chisinau, the Republic of Moldova included yielded 283 patient isolates, representing approximately one quarter of all incident culture-positive TB cases diagnosed in Moldova during the same time period. A high proportion of isolates in this sample carried mutations associated antimicrobial drug resistance (Fig. 1a). A total of 142 (50.2\%) of these isolates carried at least one mutation associated with isoniazid resistance, 131 (46.3\%) isolates were MDR by genotype (i.e. carried mutations associated with both isoniazid and rifampin resistance), and 45 (15.9\%) of all isolates were MDR by genotype and carried gyrA mutations associated with resistance to fluoroquinolones ('pre-extensively drug resistant', pre-XDR). Previous TB treatment ( $63 \%$ vs. $24 \%, P<0.01$ ), history of homelessness ( $24 \%$ vs $14 \%, P<0.05$ ), and urban residence (68\% vs $54 \%, P<0.05)$ were factors associated with MDR or XDR-TB strains. (Here we apply the pre-2021 definition of XDR-TB, i.e. $M$. tuberculosis resistant to isoniazid and rifampin as well as fluoroquinolones and at least one injectable medication, such as streptomycin.) Among the $17 \mathrm{~TB}$ cases with HIV infection, 13 were MDR or XDR.

\section{MDR-TB in Moldova is diverse but dominated by a single epidemic strain}

We next sought to understand the population structure of M. tuberculosis in our sample, and more specifically for isolates with MDR phenotypes. M. tuberculosis isolates collected during our study in Chisinau are comprised primarily of two major phylogenetic lineages of $M$. tuberculosis, Lineage 2 (L2) and Lineage 4 (L4) (Fig. 1a). There were 80 (28.3\%) strains from phylogeographic Lineage 2 and 203 (71.7\%) strains from Lineage 4 . A total of $43.8 \%$ of Lineage 4 and $52.5 \%$ of Lineage 2 isolates collected in Chisinau were either MDR or pre-XDR by genotype. Using the SNP-based classification system described by Coll et al. [12], all Lineage 2 strains were identified as belonging to sublineage 2.2.1; six different sublineages comprised the Lineage 4 isolates in our sample (Fig. 1a). The majority of the Lineage 4 isolates in this sample are from sublineage $4.2(n=113,39.9 \%$ of all 283 isolates and $55.6 \%$ of L4 isolates).

Among the 131 genotypic MDR-TB isolates (including pre$\mathrm{XDR}$ isolates), the majority ( $n=82,62.6 \%)$ belong to the genetically monomorphic Ural/4.2 clade (Fig. 1a), whereas only $19.6 \%$ of all drug-susceptible isolates ( 28 of 143 ) were from the Ural/4.2 clade. Using an adapted application of the Kolmogorov-Smirnov test that accounts for differences in 
the number of taxa in each clade (Fig. S1), we found that terminal branch lengths were significantly shorter for Ural/4.2 isolates when compared to Chisinau isolates in Lineages 2.2 and 4.1. These results are consistent with recent and/or more frequent transmission of a single highly monomorphic strain (Figs $1 \mathrm{~b}$ and $\mathrm{S} 1$ ), although this analysis is potentially subject to sampling bias. Terminal branch lengths for Ural/ 4.2 isolates were numerically smaller than those for Lineage 4.3 and 4.8 isolates, but this difference was not statistically significant, potentially due to the smaller number of isolates from the latter two lineages. Patients harbouring Ural/4.2 strains were more likely to have reported previous TB treatment when compared to other non-Ural/4.2 (62 vs $37 \%, P<0.01$ ).

Among 77 total participants from Chisinau for which there is more than one isolate with available WGS data, we identified eight participants who had discordant drug susceptibility genotypic profiles between serial isolates (Fig. S2). Five of these participants had serial isolates with discordant lineage assignments and large SNP differences compared to their initial isolates (Fig. S2a-e), suggestive of either secondary infection with a different strain of M. tuberculosis following initial culture collection or an initial polyclonal infection with subsequent selection for the more drug-resistant isolate during anti-TB therapy. Among these four participants, MDR Ural/4.2 M. tuberculosis strains were recovered as the later, discordant isolate in two individuals. Three other participants (Fig. S2f-h) had genotypic drug susceptibility profiles and inter-isolate SNP differences consistent with de novo acquisition of new drug resistance mutations between serial isolates. Of the remaining 69 participants with more than one isolate and concordant genotypic drug susceptibility profiles, none had discordant SNP-based lineage assignments, i.e. there was no evidence of polyclonal or secondary infection among participants with consistent drug susceptibility profiles across serial isolates.

\section{Prior TB treatment is associated with clustered transmission}

We next assessed the extent of genomic clustering within our dataset, using two separate metrics: [1] (1) SNP threshold, where isolates diverging by no more than ten SNPs (with SNP differences calculated using all SNPs, including those associated with drug resistance) were considered clustered; and(2) a probabilistic approach that incorporates the SNP differences and information about case timing, the molecular clock rate, and transmission processes [13]. Both methods were similar, indicating 36.2 and $36.9 \%$ were clustered by the cutoff and probabilistic approach, respectively. Previous TB treatment was associated ( $54 \%$ vs. $37 \%, P<0.01)$ with clustering by both methods.

\section{Ural/4.2 strains are found across Eastern Europe}

To place our analyses in a broader phylogenetic and geographic context, we combined sequence data from $n=283$ unique patient isolates collected in Chisinau with a larger set of publicly available $M$. tuberculosis genomes $(n=1168)$ from Eastern Europe (Fig. 2). Isolates with genotypic multidrug resistance were widely prevalent in this collection, comprising $63.5 \%$ of all isolates in the dataset (Fig. 2); $32.5 \%$ of all isolates $(n=371)$ carry a gyrA mutation associated with fluoroquinolone resistance. $M$. tuberculosis clades generally distributed across multiple countries, with the exception of Ural/4.2, which is largely comprised of Moldovan isolates collected during our study. In total, 335 of 1451 isolates (23.1\%) in this collection were identified as lineage Ural/4.2, of which 275 (82.1\%) were collected in Moldova. Excluding samples collected during our study in Chisinau, Ural/4.2 isolates comprise $14.7 \%$ (172 of 1168) of the larger collection of isolates from Eastern Europe. We found that 112 of the $172(65.1 \%)$ Ural/4.2 isolates from the regional collection of samples were collected in Moldova.

\section{Phylogenetic reconstruction indicates emergence of MDR Ural/4.2}

To examine the timeline over which the Ural/4.2 outbreak strain acquired multidrug resistance, we estimated time to most recent common ancestor (TMRCA) for clades carrying canonical drug resistance mutations in inhA (including its associated promoter region) and $k a t G$ (associated with resistance to isoniazid), $r p o B$ (associated with resistance to rifampin), and gyrA (associated with resistance to fluoroquinolones) on the phylogenetic tree (Fig. 3). We define the emergence date for MDR Ural/4.2 as the TMCRA isolates carrying the strain-specific $r p o B$ mutation $S 450 \mathrm{~L}$, which confers resistance to rifampin and the MDR phenotype. We note that undetected homoplasic acquisition of drug resistance mutations within clades can potentially bias these estimates toward longer TMRCA (earlier dates of emergence) and, where this possibility is suggested by the phylogenetic tree, we report ages for multiple nodes representing potential most recent common ancestors for a given mutation (Fig. 3a). We estimated that Ural/4.2 evolved resistance to isoniazid (via acquisition of kat $G$ S315T) in approximately 1984 (Node A, 95\% HPD: 1970-1995) and acquired the rpoB S450L mutation, conferring resistance to rifampin, at some time between 1983-2000 (Node J, TMRCA: 1993). A candidate compensatory mutation in $r p o C \mathrm{~V} 483 \mathrm{G}$ arose coincident with $r p o B$ S450L (Fig. S3). Additional Ural/4.2 mutations in $r p s L$ K88R (conferring resistance to streptomycin) and inhA promoter -C15T (conferring resistance to isoniazid) were acquired in 1984 (Node A, 95\% HPD: 1970-1995) and 1987 (Node B, 95\% HPD: 1976-1997), respectively. A variety of gyrA mutations are observed across Ural/4.2 isolates, suggesting that MDR-TB Ural/4.2 strains independently acquired $g y r A$ mutations multiple times, subsequent to the acquisition of mutations associated with isoniazid and rifampin resistance. Thus, phylogenetic analysis suggests MDR Ural/4.2 strains emerged in the early 1990s acquiring multiple resistance mutations (i.e. in $k a t G$, inh $A$ promoter, $r p o B$ ) prior to expansion as a highly drug resistant clone. We found no known resistance-associated polymorphisms in genes previously implicated in resistance to bedaquiline (Rv0678, atpE, and $p e p Q[14])$ among isolates from Chisinau. Lineage 2.2.1 isolates collected in Chisinau all exhibited a characteristic T2A mutation in es $x W$, which has 


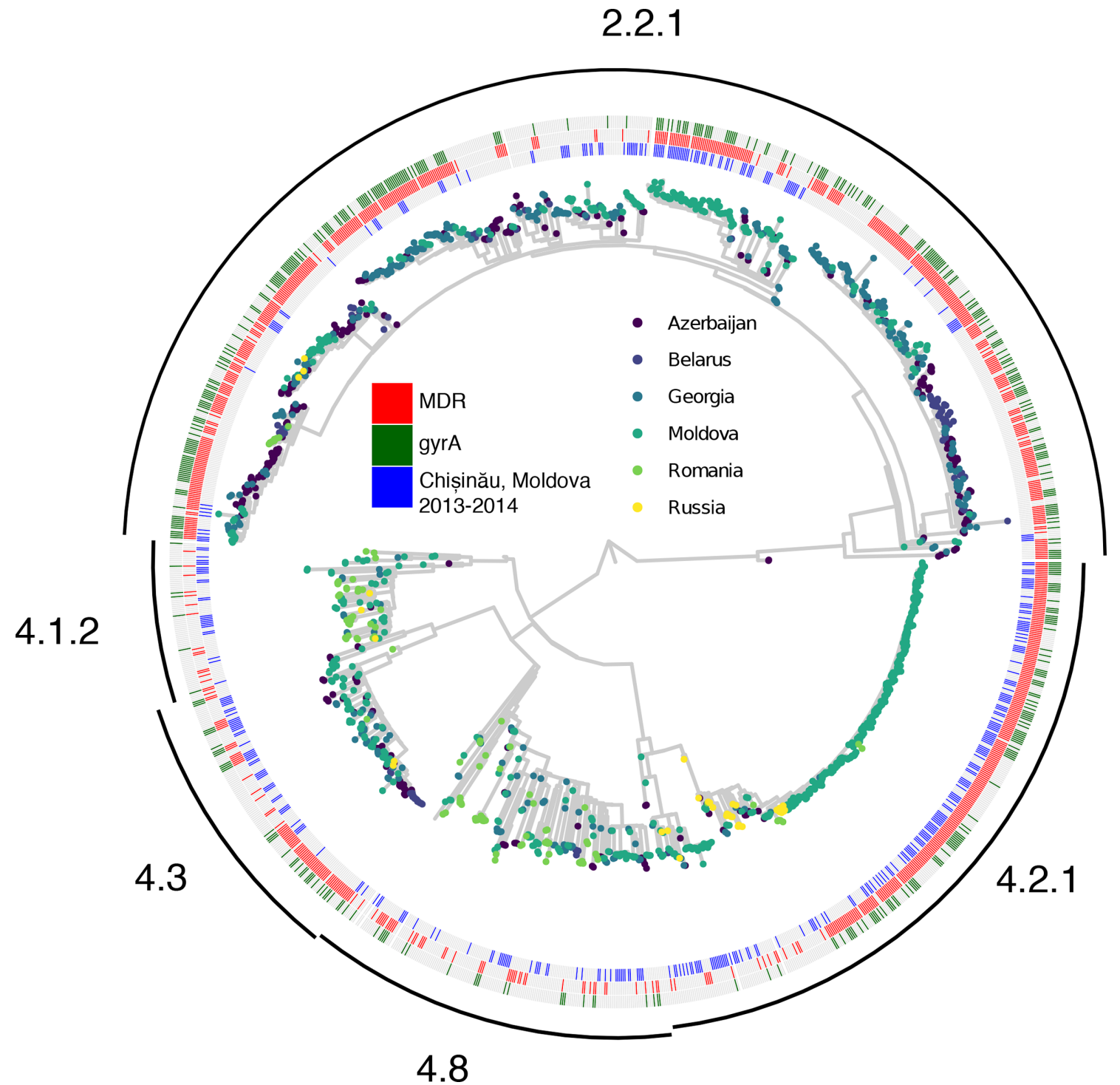

Fig. 2. Bayesian phylogenetic reconstruction for $n=1451$ M. tuberculosis isolates from Eastern Europe. Tips of the phylogeny are coloured by the country of origin for each isolate. Annotations denote M. tuberculosis sequences collected in Chisinau during this study (inner ring, red) versus sequences from prior studies (inner ring, grey), sequences with genotypic multidrug-resistance (middle ring, blue), and sequences with any fluoroquinolone resistance-associated gyrA polymorphism (outer ring, blue). The phylogenetic tree was estimated using the IQtree and timetree tools in Nextstrain Augur 6.2.0 [11]. Additional details on this group of samples is available in Table S1. Clades of the tree are annotated with SNP barcode-based sublineage assignments [12].

been associated with increased transmissibility in Lineage 2 $M t b$ strains [15]; a subset of Lineage 2.2.1 isolates also carried a frameshift mutation in $k d p D$ (c.2541_2542delCA) that has been associated with increased virulence [16]. Neither esxW T2A or the $k d p D$ frameshift mutation were observed among Ural/4.2 isolates in Chisinau (Fig. S4).

Fig. 3(c) shows the estimated $M$. tuberculosis bacterial population size for Ural/4.2 isolates over time inferred from Bayesian phylogenetic reconstruction using an exponential demographic model (the best-supported model in our analysis, see Supplementary methods). Using this model, we observe substantial expansion of the M. tuberculosis effective population size $\left(N_{e}\right)$ starting between 1995 and 2000 (Fig. 3c). To further test the hypothesis that Ural/4.2 M. tuberculosis bacterial population size expanded recently, we used nonparametric Bayesian skyline analysis to estimate the historical size of Ural/4.2 population. Consistent with the exponential demographic model, we observed expansion in the effective population size of Ural/4.2 strains starting on or around the year 2000 (Fig. S5). Model testing for Bayesian phylogenetic analysis, which favours exponential growth as the bestperforming demographic model and the aforementioned Bayesian Skyline as the second-best model (Table S2), provides additional supporting evidence for recent prior population 
(a)

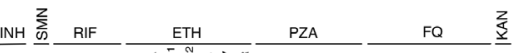
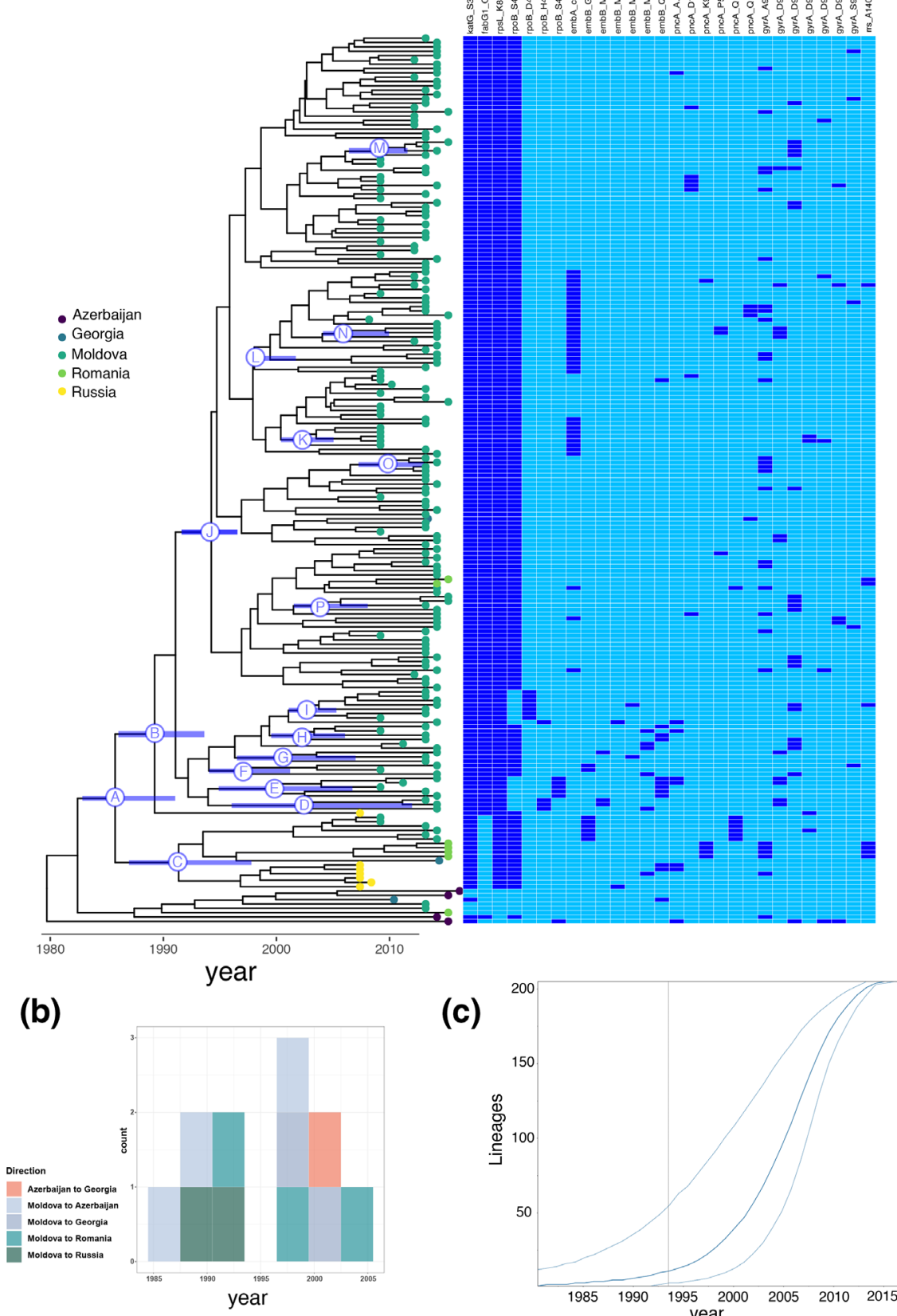

(c)

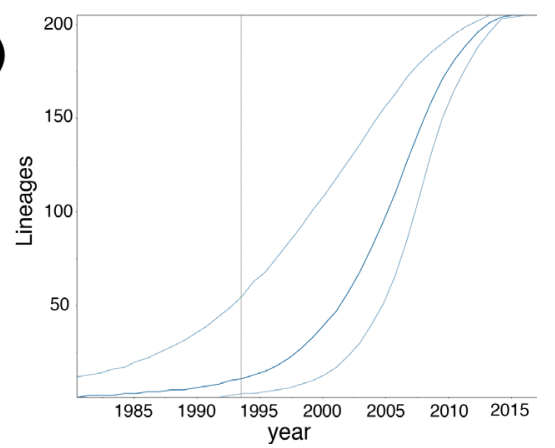

Fig. 3. Bayesian phylogenetic reconstruction with discrete trait analysis for country of origin for $n=205 \mathrm{M}$. tuberculosis isolates from the Ural/4.2.1 lineage, including the multidrug resistant outbreak strain and closely related isolates. (a) Phylogenetic reconstruction and estimated TMRCA for isolates carrying known M. tuberculosis drug resistance mutations. Nodes corresponding to most recent common ancestor for relevant drug resistance mutations are labelled A-0, with the $95 \%$ highest probability density for node age displayed the blue bar on each labelled node. Tips are labelled with the country of origin for each isolate (Azerbaijan, Georgia, Moldova, Romania, and Russia). Median node ages and 95\%HPD (listed in parentheses are as follows: A: katG S315T 1984 (1970-1995); B: fabG C [15]T 1987 (1976-1997); C: rpoB S450L 1990 (1979-1999); D: rpoB H455N 2001 (1992-2009); E: rpoB S450W 1998 (1990-2005) F: rpoB S450L 1995 (1986-2003); G: rpoB S450L 1999 (1991-2007); H: rpoB S450L 2011 (1995-2006); I: rpoB D435Y 2001 (1996-2006); J: rpoB S450L 1993 (1983-2000); K: embA C(16T) 2001 (1996-2006) L: embA C(16T) 1996 (1989-2003); M: gyrA D94G 2008 (2005-2011); N: gyrA D94A 2005 (1999-2010; 0: gyrA A90V 2009 (2005-2011). (b) Discrete trait analysis for migration events. Boxes show counts for estimated number of migration events between countries by year and are coloured according to pairs of sending and receiving countries. (c) Estimated effective population size over time with exponential demographic model for Bayesian phylogenetic reconstruction. Outer lines show the $95 \%$ highest posterior density interval for the effective population size. 
growth, consistent with relative increases in overall transmission, for the Ural/4.2 outbreak clade. Taken together, our results indicate that MDR Ural/4.2 clade emerged in the early 1990s achieving sustained transmission in the early 2000 s with resultant increases in bacterial population size. At the time of this study, almost all TB patients initiated treatment as inpatients, and average length of hospitalization exceeded 2 months as recently as 2016 .

\section{Geographic origin and regional dispersal of MDR Ural/4.2 strains}

Next, we performed analyses to formally examine the phylogeographic history of Ural/4.2 strains in Eastern Europe, treating sampling location (country) as a discrete trait. Applying DTA, the geographic location of the MRCA for Ural/4.2 sequences was estimated to be Moldova with posterior probability $=0.66$, although this inference may be influenced by unsampled locations or regions (Fig. 3b). To investigate the migration history of Ural/4.2 over time, we analysed the inferred load and direction of migration over time (see Supplementary Information). Inferred migration events of Ural/4.2 strains indicate drug-resistant and MDR Ural/4.2 strains were exported from Moldova to neighbouring countries (Azerbaijan, Georgia Romania and Russia) from mid-1980s onwards, with additional estimated migration events between Azerbaijan and Georgia after the year 2000.

\section{DISCUSSION}

Countries in Eastern Europe record the highest incidence rates of drug-resistant tuberculosis globally. Here, we report transmission of a specific, epidemiologically successful strain of $M$. tuberculosis (MDR Ural/4.2) as the most frequent cause of MDR-TB in a cross-sectional cohort of patients in Chisinau, Moldova. Our results indicate that this drug-resistant strain emerged in the early 1990s and underwent marked population expansion after achieving sustained transmission in the early 2000s. Crudu et al. previously reported that nosocomial re-infection resulting in MDR-TB in the Republic of Moldova was often attributable to a MDR-TB Ural/4.2 strain [17]. Since this study focused only on Chisinau, we do not yet know the extent to which this strain circulates within the rest of Moldova. However, we do find limited phylogenetic evidence indicating recent exportation of MDR Ural/4.2 from Moldova to neighbouring countries in Eastern Europe, underscoring the need for regional coordination in TB surveillance and control.

There is increasing evidence that transmission of existing drug-resistant strains is the primary driver of new infections in most drug-resistant TB epidemics $[3,5]$. We find that despite accounting for only a small proportion of the drug susceptible bacillary population (19.6\%), Ural/4.2 strains are heavily enriched among MDR and pre-XDR groups of isolates (62.6\%). Short terminal branch lengths indicate that this enrichment is most likely driven by recent or more frequent transmission. Alternatively, shorter branch lengths observed in the Ural/4.2 clade could be attributable to geographic or other kinds of sampling bias, if such bias resulted in preferential sampling of clustered Ural/4.2 isolates and/or under-sampling of isolates from other clades, but it is difficult to measure or account for this hypothetical source of bias. Notably, our sample of isolates from Chisinau is enriched for Ural/4.2 strains, despite coendemnicity with Lineage 2.2.1 strains carrying variants associated with increased virulence and/or transmissibility (esxW T2A and the c.2541_2542delCA frameshift mutation in $k d p D)[15,16]$.

Consistent with recent findings from Sinkov et al. [7], we find that MDR-TB Ural/4.2 strains emerged in the early 1990s, approximately at the time of Moldovan independence from the Soviet Union. This period was characterized by a decade of economic turmoil, mass incarceration, underfunded public health programmes, and unreliable TB drug supplies [18]. This complex interplay between socio-economic conditions and poor TB case management likely created conditions conducive to the evolution of MDR and other drug-resistant M. tuberculosis strains, although direct association between these factors and the emergence of MDR-TB Ural/4.2 is limited by the ecological nature of this study.

We infer from Bayesian phylogenetic analysis that Ural/4.2 has undergone substantial and relatively recent $M$. tuberculosis bacterial population expansion. Using both an exponential demographic model and the non-parametric Bayesian Skyline method (two of the best-performing demographic models in our analysis per marginal likelihood estimation), we observe evidence of substantial increases in the Ural/4.2 bacterial population from the year 2000 onward. Starting in the early 2000s and continuing until 2014, TB control policy in Moldova recommended that all TB patients be hospitalized during the intensive phase of therapy and MDR and non-MDR patients were separated only after phenotypic DST results were available [19]. We find that MDR-TB Ural/4.2 strains were more likely recovered from individuals with previous TB history and that Ural/4.2 strains were isolated from approximately half of all follow-up discordant serial isolates, suggesting a role for exogenous reinfection during anti-TB therapy. This phenomenon, i.e. secondary or polyclonal infection identified via genotyping of serial isolates, is observed in other cross-sectional M. tuberculosis genomic surveillance studies where an epidemiologically-successful drug-resistant strain is circulating in the community $[20,21]$.

There is concern that MDR and pre-XDR M. tuberculosis strains can disseminate beyond international borders and seed neighbouring and more distant countries [22, 23]. Our analysis indicates that Ural/4.2 was exported from Moldova to at least four countries in Eastern Europe between $\sim 1985$ and 2005 (Fig. 3c). Although we document multiple cross-border strain migration events, extensive regional dissemination appears to be limited, consistent with prior studies on the global distribution of drug-resistant TB strains [24]. We acknowledge that our collection of Ural/4.2 isolates is prominently over-sampled for isolates collected 
in Chisinau and under-sampled for isolates from the rest of the country and from countries other than Moldova, and that this is an important limitation on our ability to infer historical migration events from the available data, although expect that this limitation would result in bias toward inferring fewer migration events overall. Likewise, this locally-collected sample is expected to yield larger proportions of clustered infections, given that individuals living within a local catchment area are more likely be linked by clustered transmission compared to those living across wider geographic areas [25]. Our findings indicate regional dissemination may be nascent and aggressive approaches to contain MDR Ural/4.2 strains locally may prevent further cross border events.

Taken together, our results underscore the intersection of socio-economic factors and public health policy in shaping drug resistant TB epidemics. Our findings highlight how large systematic shortcomings in clinical, public health and social programmes can facilitate the emergence and transmission of drug-resistant TB. Clustered transmission and history of previously treated $\mathrm{TB}$ were associated with Ural/4.2 MDR-TB infection in our study, indicating that nosocomial transmission may have contributed to the emergence and spread of this strain in Moldova. To avoid widespread regional transmission of highly drug resistant strains, adequate resources for local containment and regional control efforts will be required. Our study supports regional cooperation and coordination in TB control $[26,27]$ and underscores the need for regional public access data that systematically incorporates epidemiological and pathogen information.

\section{Funding information}

This study was funded by the United States National Institutes of Health (NIH T32AI007061 to TSB), the International Union Against Tuberculosis and Lung Disease, and the United States Agency for International Development. None of the funding bodies had any role in the design of the study or collection, analysis, and interpretation of data or in writing the manuscript.

\section{Acknowledgements}

We would like to acknowledge in kind support from the American Museum of Natural History for providing computational resources.

\section{Author contributions}

T. S. B., V. E., O. B. and M. O., planned and conducted the data analysis and co-wrote the manuscript (formal analysis, writing-original draft preparation and editing). N. S., planned and conducted data analysis (formal analysis). J. S., developed analytical methods used for strain clustering (methodology, software). C. C., supervised data analysis and co-wrote the manuscript (supervision, writing-reviewing and editing). S. A., E. N. and N. C., supervised and conducted laboratory analysis (supervision, investigation). V. C., T. H. C. and B. M., designed the study, supervised laboratory work and data analysis, and co-wrote the manuscript (conceptualization, supervision, writing-original draft preparation and editing, writing-reviewing and editing).

\section{Conflicts of interest}

The authors declare that there are no conflicts of interest.

\section{Ethical statement}

The study protocol was approved by the Institutional Ethical Committee for Research of Phthisiopneumology Institute, Chisinau, Republic of Moldova (Approval Oct 2012), Partners Human Research Committee
(2012-P-001904/1), Yale University Human Investigation Committee (IRB number: 1409014628), and Columbia University Medical Centre (Not Human Subjects Research Under 45 CFR 46).

References

1. endTB. 2020. www.endtb.org [accessed 01 Feb 2021].

2. Houben R, Menzies NA, Sumner T, Huynh GH, Arinaminpathy $N$, et al. Feasibility of achieving the 2025 WHO global tuberculosis targets in South Africa, China, and India: a combined analysis of 11 mathematical models. Lancet Glob Health 2016;4:e806-e815.

3. Shah NS, Auld SC, Brust JC, Mathema B, Ismail N, et al. Transmission of extensively drug-resistant Tuberculosis in South Africa. $N$ Engl J Med 2017;376:243-253.

4. Yang C, Luo T, Shen X, Wu J, Gan M, et al. Transmission of multidrugresistant Mycobacterium tuberculosis in Shanghai, China: a retrospective observational study using whole-genome sequencing and epidemiological investigation. Lancet Infect Dis 2017;17:275-284.

5. Brown TS, Challagundla L, Baugh EH, Omar SV, Mustaev A, et al. Predetection history of extensively drug-resistant tuberculosis in KwaZuluNatal, South Africa. Proc Natl Acad Sci U S A 2019;116:23284-23291.

6. Toungoussova OS, Bjune G, Caugant DA. Epidemic of tuberculosis in the former Soviet Union: social and biological reasons. Tuberculosis (Edinb) 2006:86:1-10.

7. Sinkov V, Ogarkov O, Mokrousov I, Bukin Y, Zhdanova S, et al. New epidemic cluster of pre-extensively drug resistant isolates of Mycobacterium tuberculosis Ural family emerging in Eastern Europe. BMC Genomics 2018;19:762.

8. Rosenthal A, Gabrielian A, Engle E, Hurt DE, Alexandru S, et al. The TB portals: An open-access, web-based platform for global drugresistant-tuberculosis data sharing and analysis. J Clin Microbiol 2017:55:3267-3282.

9. Bouckaert RR, Drummond AJ. bModelTest: Bayesian phylogenetic site model averaging and model comparison. BMC Evol Biol 2017:17:42.

10. Suchard MA, Lemey $P$, Baele G, Ayres DL, Drummond AJ, et al. Bayesian phylogenetic and phylodynamic data integration using BEAST 1.10. Virus Evol 2018;4:vey016.

11. Hadfield J, Megill C, Bell SM, Huddleston J, Potter B, et al. Nextstrain: real-time tracking of pathogen evolution. Bioinformatics 2018:34:4121-4123.

12. Coll F, McNerney R, Guerra-Assuncao JA, Glynn JR, Perdigao J, et al. A robust SNP barcode for typing Mycobacterium tuberculosis complex strains. Nat Commun 2014;5:4812.

13. Stimson J, Gardy J, Mathema B, Crudu V, Cohen T, et al. Beyond the SNP threshold: Identifying outbreak clusters using inferred transmissions. Mol Biol Evol 2019;36:587-603.

14. Nguyen TVA, Anthony RM, Banuls AL, Nguyen TVA, DH V, et al. Bedaquiline resistance: Its emergence, mechanism, and prevention. Clin Infect Dis 2018;66:1625-1630.

15. Holt KE, McAdam P, Thai PVK, Thuong NTT, Ha DTM, et al. Frequent transmission of the Mycobacterium tuberculosis Beijing lineage and positive selection for the EsxW Beijing variant in Vietnam. Nat Genet 2018;50:849-856.

16. Parish T, Smith DA, Kendall S, Casali N, Bancroft GJ, et al. Deletion of two-component regulatory systems increases the virulence of Mycobacterium tuberculosis. Infect Immun 2003;71:1134-1140.

17. Crudu V, Merker M, Lange C, Noroc E, Romancenco E, et al. Nosocomial transmission of multidrug-resistant tuberculosis. Int J Tuberc Lung Dis 2015;19:1520-1523.

18. Dávalos ME, Nguyen TC, Matytsin M. Poverty Reduction and Shared Prosperity in Moldova: Progress and Prospects. Washington, DC: World Bank Group; 2016.

19. Cercone J. Technical assistance to the ministry of health and the national TB program in investment planning and improvement of TB service delivery system in the Republic of Moldova: Consultancy report. Miami: Sanigest, 2013 
20. Crudu V, Romancenco E, Noroc E, Alexandru S, Niemann S, et al. Beijing and H4/Ural genotypes of M Tuberculosis are predominating among M/ XDR-TB patients in Moldova. Int J Tuberc Lung Dis 2014:18.

21. O'Donnell MR, Larsen MH, Brown TS, Jain P, Munsamy V, et al. Early detection of emergent extensively drug-resistant Tuberculosis by flow cytometry-based phenotyping and whole-genome sequencing. Antimicrob Agents Chemother 2019:63.

22. Cooke GS, Beaton RK, Lessells RJ, John L, Ashworth S, et al. International spread of MDR TB from Tugela Ferry, South Africa. Emerg Infect Dis 2011:17:2035-2037.

23. Coscolla M, Barry PM, Oeltmann JE, Koshinsky H, Shaw $T$, et al. Genomic epidemiology of multidrug-resistant Mycobacterium tuberculosis during transcontinental spread. J Infect Dis 2015;212:302-310.
24. Brynildsrud OB, Pepperell CS, Suffys P, Grandjean L, Monteserin J, et al. Global expansion of Mycobacterium tuberculosis lineage 4 shaped by colonial migration and local adaptation. Sci Adv 2018:4:eaat5869.

25. Glynn JR, Vynnycky E, Fine PE. Influence of sampling on estimates of clustering and recent transmission of Mycobacterium tuberculosis derived from DNA fingerprinting techniques. Am J Epidemiol 1999:149:366-371.

26. Drobniewski F, Ehsani S, Dara M. Fighting drug-resistant tuberculosis in eastern Europe. Lancet Infect Dis 2017;17:691-692.

27. Jagielski T. Partnership to Fight Against TBiC, Eastern E. FATE: the new partnership to Fight Against TB in Central and Eastern Europe. Lancet Infect Dis 2017:17:363.

\section{Five reasons to publish your next article with a Microbiology Society journal}

1. The Microbiology Society is a not-for-profit organization.

2. We offer fast and rigorous peer review - average time to first decision is 4-6 weeks.

3. Our journals have a global readership with subscriptions held in research institutions around the world.

4. $80 \%$ of our authors rate our submission process as 'excellent' or 'very good'.

5. Your article will be published on an interactive journal platform with advanced metrics.

Find out more and submit your article at microbiologyresearch.org. 